\title{
Disulfide Modified IgG1: An Investigation of Biophysical Profile and Clinically Relevant Fc Interactions
}

\author{
Calise Bahou ${ }^{\mathrm{ac}}$, Elizabeth A. Love ${ }^{c}$, Siobhán Leonard ${ }^{\mathrm{c}}$, Richard J. Spears ${ }^{\mathrm{a}}$, Antoine Maruani ${ }^{\mathrm{a}}$, Kathryn \\ Armour $^{c}$, James R. Baker ${ }^{\mathrm{a} *}$, Vijay Chudasama ${ }^{\mathrm{ab} *}$ \\ ${ }^{a}$ Department of Chemistry, University College London, 20 Gordon Street, WC1H OAJ, London, UK. \\ ${ }^{b}$ Research Institute for Medicines (iMed.ULisboa), Faculty of Pharmacy, Universidade de Lisboa, Lisbon, Portugal. \\ ${ }^{c}$ LifeArc, Accelerator Building, SBC Open Innovation Campus, SG1 2FX, Stevenage, UK.
}

*E-mails: j.r.baker@ucl.ac.uk,v.chudasama@ucl.ac.uk 


\section{Abstract}

Modification of immunoglobulin $\mathrm{G}(\mathrm{IgG}) 1$ proteins in cancer treatment is a rapidly growing field of research. Antibody-drug conjugates (ADCs) exploit the targeted nature of this immunotherapy by conjugating highly potent drugs to antibodies, allowing for effective transport of cargo(s) to cancerous cells. Of the many bioconjugation strategies now available for the formation of highly homogenous ADCs, disulfide modification is considered an effective, low-cost and widely accepted method for modifying IgG1s for improved clinical benefit. However, little is known about how disulfide modification impacts clinically relevant fragment crystallisable $(\mathrm{Fc})$ region interactions. Although often overlooked as a secondary ADC function, Fc interactions could prove key in rational design of cancer cell-targeting ADCs through consideration of potent mechanisms such as antibodydependant cellular cytotoxicity (ADCC). This work explores different IgG1 disulfide modification techniques and the effect they have on quantifiable secondary IgG1 Fc interactions (e.g. CD16a and FcRn). The solvent accessible disulfide residues of trastuzumab, a clinically relevant IgG1, were modified to provide a range of bioconjugates with differing amounts of inter-chain covalent linkages. It was found that by natively re-bridging the IgG1 model, all tested Fc functionalities were not significantly affected. Additionally, in non Fc-specific biophysical experiments (e.g. thermal stability/aggregation), the natively re-bridged species provided an exceptional profile, showing no significant change from the tested native antibody. Conjugates with significant disruption of the covalent connectivity of IgG1 chains resulted in a suboptimal Fc profile (CD16a kinetics or ADCC activity), in addition to sub-standard non Fc-specific attributes (thermal stability). These results advocate native disulfide re-bridging as an excellent synthetic strategy for forming homogenous IgG1 bioconjugates, with no reported negative impact on biophysical profile relative to the native antibody.

\section{Introduction}

Protein modification has become a fundamental tool in the preparation of active biomolecules for cancer treatment. ${ }^{1,2}$ More specifically, modification of native monoclonal antibodies (mAbs) allows for targeted delivery of highly potent drugs to cancer cells, in the form of antibody-drug conjugates (ADCs). ${ }^{3,4}$ To date, four ADCs have been approved for use by the US Food and Drug Administration (FDA) (Mylotarg ${ }^{\mathrm{TM}}$, Besponsa ${ }^{\mathrm{TM}}$, Kadcyla ${ }^{\mathrm{TM}}$, and Adcetris $^{\mathrm{TM}}$ ) with over 60 more currently being evaluated in clinical trials. ${ }^{5-11}$ Modification of native amino acid residues has been utilised for the majority of the clinically relevant ADCs thus far, likely owing to the lower costs of manufacture. Typically, the natural amino acids targeted for site-selective modification are lysine and cysteine residues - for their highly nucleophilic $\varepsilon$-amino and thiol groups respectively. ${ }^{2}$ Cysteine residues are often preferred in the synthesis of homogenous antibody conjugates as lysine modification reagents (e.g. $N$-hydroxysuccinimide esters) randomly conjugate over $c a .40$ lysine residues on a typical IgG1. ${ }^{12}$ This can result in a heterogeneous mixture of conjugates with a poorly defined pharmacokinetic profile (e.g. clearance rates, binding activities etc.), leading to a narrower therapeutic window when compared to an analogous homogeneous conjugate. ${ }^{13-16}$ Due to the low natural abundance of cysteine in IgG1 antibodies (i.e. only available through reduction of solvent accessible inter-chain disulfides), the highly reactive thiol moiety is an attractive option in the formation of homogenous ADCs. ${ }^{17}$ By reducing the four solvent accessible disulfide bonds (2), it is possible to react the liberated thiol groups in a site-selective fashion to obtain an ADC (3) with up to eight payloads (thiol capping - Figure 1a). However, depending on the payload employed by the desired ADC, lower drug-to-antibody ratios (DARs) are often preferred. A DAR of two to four is typically selected in ADC design due to the optimal balance between renal clearance and potency being in this range, generally. ${ }^{18}$ Consequently, capping strategies often target lower DARs by 
reducing an average of 2 disulfides (4, Figure $1 \mathrm{~b}$ ), although heterogeneity issues arise from reduction of more/less disulfides than intended (5, Figure $1 \mathrm{~b}) .^{9,19}$

Following reduction of the solvent accessible disulfides, it is also possible to re-bridge with a chemical modification to reform the IgG covalent linkage (6, disulfide re-bridging - Figure $1 \mathrm{c}) \cdot{ }^{20-22}$ Highly homogenous ADCs with a DAR of two or four have been obtained via this strategy, and these show no decrease in antigen-binding and no decrease in circulation half-life. ${ }^{23-25}$ However, it has been observed that these disulfide residues can re-bridge in a non-native fashion, resulting in the "half-antibody" species 7, thus yielding a mixture of two antibody conjugates (Figure 1c). ${ }^{26,27 a, 27 b}$ Currently there have been no studies to suggest this "half-antibody" species (7) negatively affects the profile of the antibody. Reagents and conditions to reduce the amount of this species in bioconjugate samples have however been successfully developed, generating a more homogeneous $\mathrm{ADC}^{20,21}$

a)

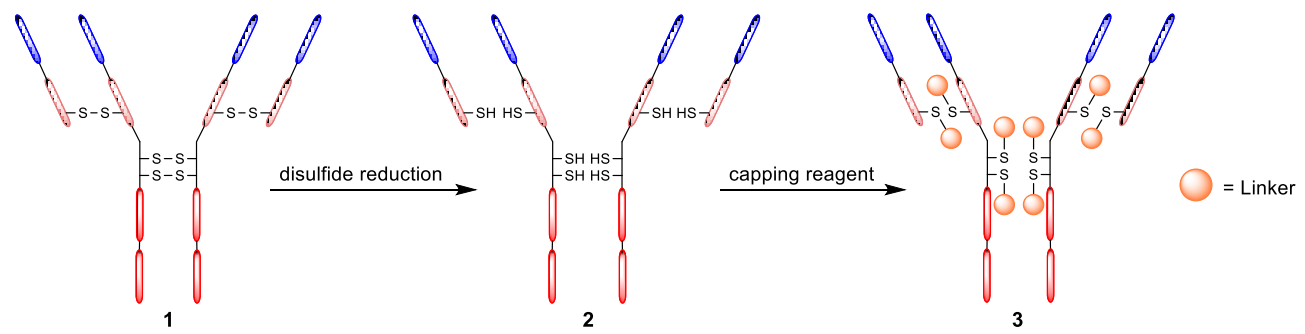

b)

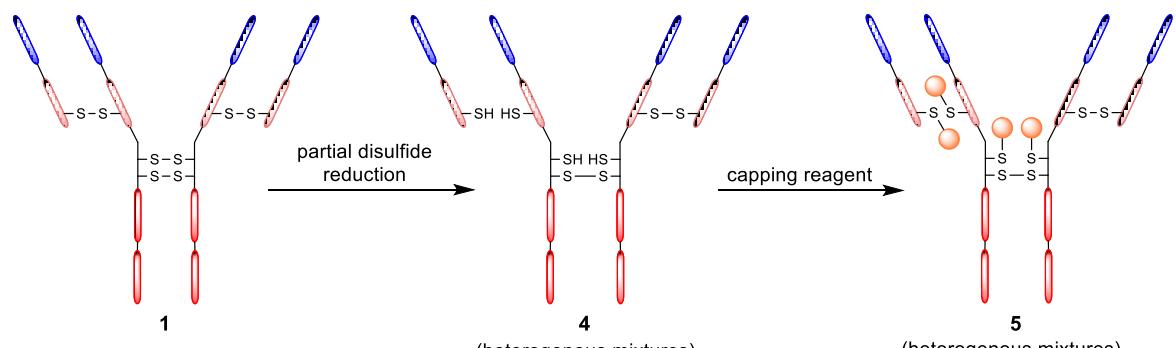

c)

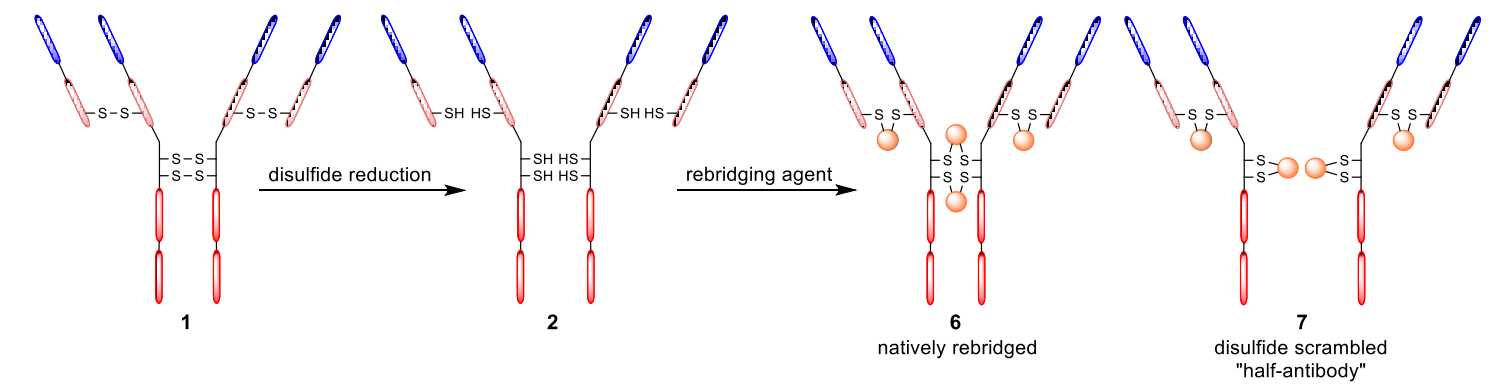

Figure 1 -Disulfide modification via reduction of solvent accessible IgG1 disulfides subsequent reaction of liberated thiols: a) reduction of all available disulfides and reaction of each liberated thiol with capping species, b) partial reduction of disulfide residues and reaction of each liberated thiol with capping species, c) reduction of all available disulfides and re-bridging of two liberated thiols with re-bridging species.

Native antibody modification is a powerful tool in the synthesis of ADCs; however, it is also considered a highly intricate process with a multitude of antibody functions to preserve postconjugation. ${ }^{28-30}$ Although antigen-binding is defined for each of the conjugation strategies outlined above, Fc activity is often overlooked as a secondary interaction. Antibodies targeting cancer cells directly can benefit from a potent secondary mechanism such as antibody-dependant cellular 
cytotoxicity (ADCC) (e.g. when an antibody internalises poorly). ${ }^{31}$ Antibodies with potent Fc function may be selected for this reason, as a platform for synthesising an effective ADC (e.g. trastuzumab and brentuximab in the synthesis of Kadcyla ${ }^{\mathrm{TM}}$ and Adectris ${ }^{\mathrm{TM}}$ respectively). ${ }^{9,32}$ It is therefore favourable to develop a conjugation technology which avoids negatively impacting these valuable IgG1 functionalities. ${ }^{29,31}$

In this work, the influence of disulfide modification on IgG1 Fc activity was explored. For this investigation, a small library of IgG1 antibody conjugates was synthesised with varying degrees of covalent integrity between antibody chains. The disulfide modified conjugates considered were as follows: natively re-bridged 6; disulfide scrambled 7; thiol capped with a maleimide-to-antibody ratio of 4 (MAR 4) 5; thiol capped with a maleimide-to-antibody ratio of 8 (MAR 8) 3. For a comparative model, the IgG1 antibody trastuzumab 8 was selected as a good representation of an IgG1 platform that has been modified for increased clinical benefit (e.g. as observed in the FDA-approved ADC $\left.\mathrm{Kadcyla}^{\mathrm{TM}}\right)^{7}{ }^{7}$ Conjugation to the disulfide residues of trastuzumab has also been well documented, using a library of conjugation reagents. ${ }^{20,23-25,27 a, 33,34}$ The conjugates synthesised for this work were modified with small $(<200 \mathrm{Da})$, chemically similar linkers so that any observable effect on the characteristics of the antibody conjugates could be attributed to the method of disulfide modification employed. We also had to consider that the antibody conjugates compared in this study cover a range of loadings (e.g. comparison between the MAR 4 and MAR 8 conjugates) and that this large variation in hydrophobic loading would significantly affect (and potentially dominate) properties. Moreover, work presented in the literature (e.g. Gieseler and co-workers (Mol. Pharm., 2018); Jackson and co-workers (Mol. Pharm., 2015); Senter and co-workers (Nat. Biotechnol., 2015)) has highlighted how properties of ADCs can vary based on the nature of the cargo; thus by adding minimal and similar functionality to each linker we focussed on how each disulfide modification technique, at a core-level, affects properties. ${ }^{18,35 a-c}$ Two key and quantifiable Fc interactions formed the focus of our investigation, CD16a and FcRn, which provide relevant information regarding ADCC and FcRn recycling respectively. ${ }^{36}$

\section{$\underline{\text { Results }}$}

\section{Controlled manipulation of IgG1 disulfide bonds}

Trastuzumab conjugates 12-15 were synthesised ( 2 disulfide re-bridged and 2 thiol capped bioconjugates) displaying a wide range of intact disulfides and homogeneity (Figure 2). All conjugates were analysed by LC-MS, and the relative amount of the products formed under each set of conjugation conditions was approximated by densitometry analysis (SDS-PAGE, Figure 2). Trastuzumab $\mathbf{8}$ was natively re-bridged with ethyl alkyne dibromopyridazinedione $\mathbf{9}$ (diBr PD) to form a fully modified antibody conjugate 12 ( $c a$. 90\% homogenous by densitometry) by employing previously reported protocols (Figure $2 \mathrm{a}){ }^{21}$

To form a heterogeneous re-bridged trastuzumab conjugate, a new protocol was established through employment of a novel PD derivative - dichloro (DiCl) PD (10). In previous reports, it has been hypothesised that the liberated cysteines from disulfides could have a greater tendency to rebridge in a non-native conformation if fully reduced prior to addition of the re-bridging reagent..$^{20,21}$ Therefore, the inter-chain disulfides of trastuzumab were reduced for $2 \mathrm{~h}$ prior to the addition of a re-bridging agent. In addition, a less reactive re-bridging agent was sought, to increase the thiol residency times after reaction of one thiol. It was found that by using an ethyl alkyne DiCl PD (10) to re-bridge the disulfides of trastuzumab 8, a heterogeneous bioconjugate $\mathbf{1 3}$ with ca $50 \%$ of the halfantibody species was obtained (Figure $2 \mathrm{~b}$ ) (quantified by densitometry, see Figure S7 in Supporting Information). 
Two separate thiol capped trastuzumab conjugates (14 and $\mathbf{1 5}$ ) were also synthesised for comparison, demonstrating full and partial deconstruction of IgG1 disulfide covalent inter-chain links (producing maleimide-antibody ratios (MARs) of 8 and 4 respectively). For the formation of the thiol capped (MAR 8) conjugate 15, an excess of reducing agent ( 25 eq.) was added followed by an excess of $\mathrm{N}$-propargyl maleimide $\mathbf{1 1}$ (25 eq.) (Figure 2d). For the synthesis of the thiol capped (MAR 4) species 14, only 2 equivalents of reducing agent were added and left to react until completion overnight, before addition of excess maleimide ( 25 eq.) (Figure $2 \mathrm{c}$ ). To confirm a MAR of 4 (in addition to reduced MS analysis), a copper assisted azide-alkyne cycloaddition (CuAAC) reaction was carried out on the resultant conjugate to confirm a fluorescent loading of 4 (AlexaFluor ${ }^{\mathrm{TM}}-488$ ) by UV-Vis spectrometry (see Figure S11 in Supporting Information).

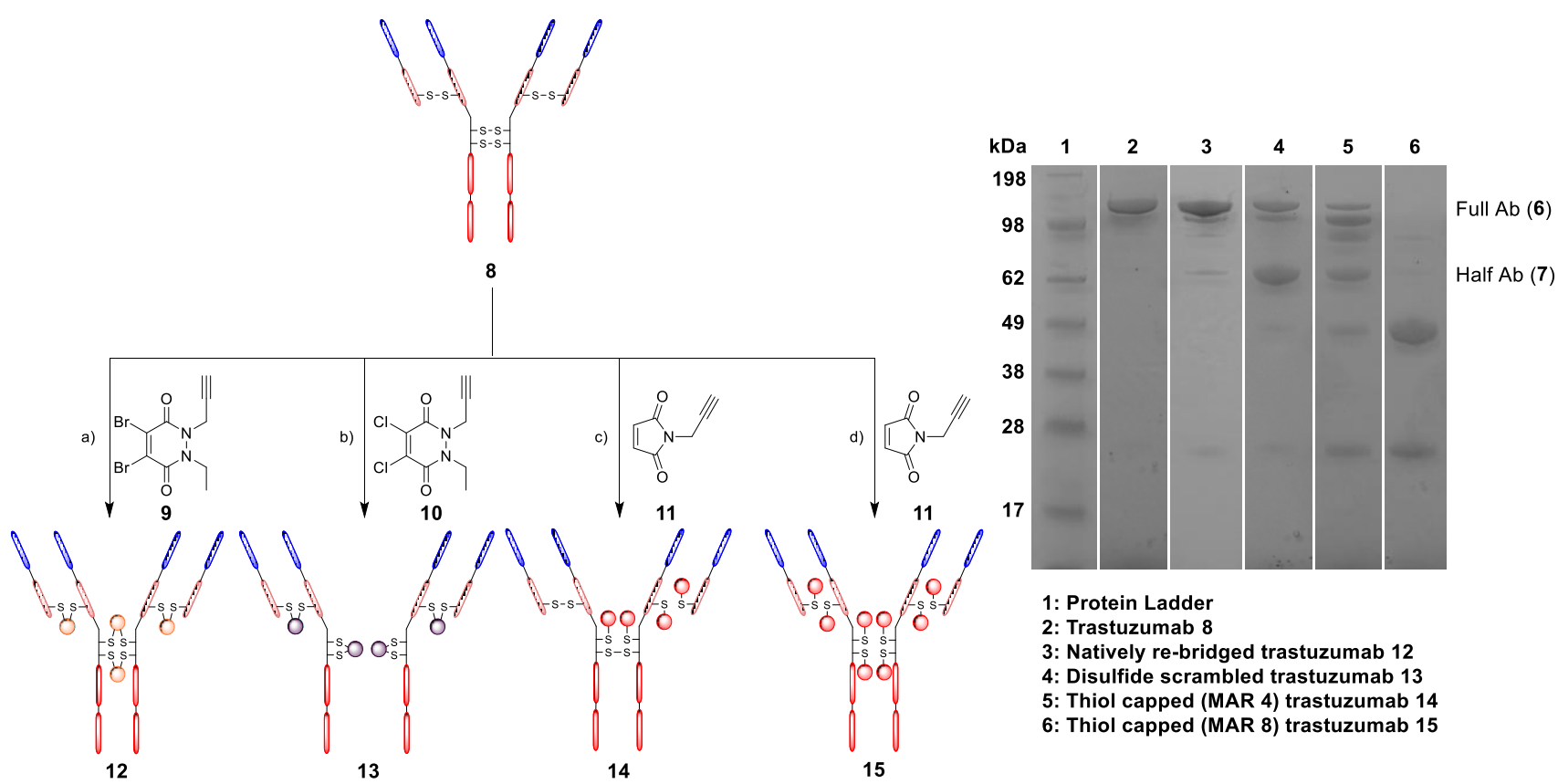

Figure 2 -Synthesis of trastuzumab conjugates, structures of most abundant species shown. All steps carried out in BBS pH 8.0, 0.25 mM EDTA: a) ethyl alkyne diBr PD 9 (20 eq.), TCEP (12 eq.), $4{ }^{\circ} \mathrm{C}$, $16 \mathrm{~h}$; b) i) TCEP ( 25 eq.), $37^{\circ} \mathrm{C}, 2 \mathrm{~h}$. ii) ethyl alkyne diCl PD 10 ( 25 eq.), $37^{\circ} \mathrm{C}, 4 \mathrm{~h}$; c) i) TCEP ( 2 eq.), $37^{\circ} \mathrm{C}, 16 \mathrm{~h}$. ii) propargyl maleimide 11 ( 25 eq.) $21^{\circ} \mathrm{C}, 16 \mathrm{~h}$; d) i) TCEP ( 25 eq.), $37^{\circ} \mathrm{C}, 2 \mathrm{~h}$. ii) propargyl maleimide 11 ( 25 eq.) $21^{\circ} \mathrm{C}, 16 \mathrm{~h}$. SDS PAGE analysis of trastuzumab 8 and trastuzumab conjugates

12-15.

\section{$\underline{\text { Trastuzumab-FcRn Assays }}$}

An FcRn bio-layer interferometry (BLI) assay was designed to mimic FcRn recycling. To demonstrate early endosomal binding conditions, the assay initially explored association and dissociation at pH 6.0 to provide an approximate, comparative $K_{D}$ for each synthesised conjugate. Additionally, mimicking release of the conjugate from $\mathrm{FcRn}$ in blood plasma, dissociation was monitored at $\mathrm{pH}$ 7.4. Due to the robust nature of the FcRn, it was recycled 3 times before discarding, showing little decrease in binding affinity. A single point concentration was selected for the comparative assay, to avoid complications with global fit analysis (stemming from the dimeric nature of the IgG1-FcRn interaction) similar to previous literature reports. ${ }^{37}$

None of the tested conjugates exhibited an approximate $K_{D}$ significantly different from the unmodified trastuzumab control 8 (Figure 3a), and no change to the dissociation rate at pH 7.4 was 
observed (Figure 3b). These results therefore suggest inter-chain disulfide residues are not likely to be involved in FcRn interactions, and that the integrity of inter-chain covalent links would not likely affect the FcRn recycling mechanism of IgG1 antibodies.
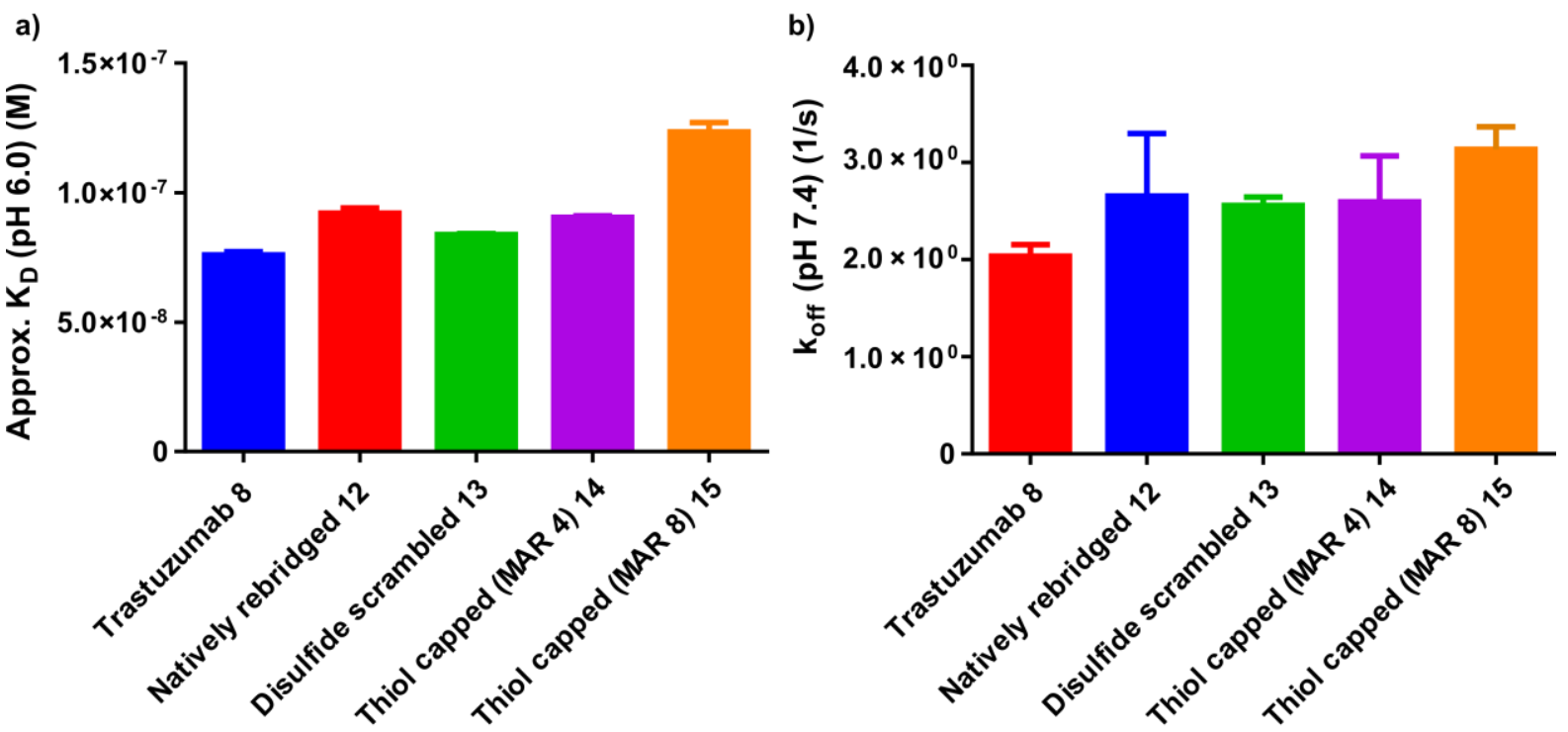

Figure 3 - Trastuzumab - FcRn BLI assay results summary. a) Average approximate $K_{D}$ values obtained at $\mathrm{pH} 6.0 ; \mathrm{b})$ Average $\mathrm{k}_{\text {off }}$ values obtained at $\mathrm{pH}$ 7.4.

\section{Trastuzumab-CD16a Assays}

A comparative BLI assay was also designed to monitor any changes in CD16a kinetics of the synthesised conjugates. The optimal platform employed streptavidin sensors loaded with biotinylated CD16a, which produced reliable results consistent with literature reports. ${ }^{33}$

The kinetic data obtained for the disulfide modified conjugates followed a general trend relating to covalent inter-chain connectivity. Importantly, natively re-bridged trastuzumab $\mathbf{1 2}$ showed very little deviation from the unmodified control 8, whilst fully thiol capped trastuzumab $\mathbf{1 5}$ had a ten-fold increase in $\mathrm{K}_{\mathrm{D}}$ (i.e. decrease in binding, Figures $4 \mathrm{a}$ and $4 \mathrm{~b}$ ). The disulfide scrambled conjugate $\mathbf{1 3}$ also appeared to yield a similar $K_{D}$ to the natively re-bridged trastuzumab $\mathbf{1 2}$, whilst the partially capped trastuzumab 14 afforded a marginally higher, yet reproducible, $K_{D}$ (Figures $4 a$ and $4 b$ ). These results highlight the native disulfide re-bridging strategy to have little to no effect on CD16a $K_{D}$ values. Furthermore, the general trend observed between these conjugates suggests intact IgG1 inter-chain covalent links may play a significant role in IgG1-CD16a kinetics. 
a)

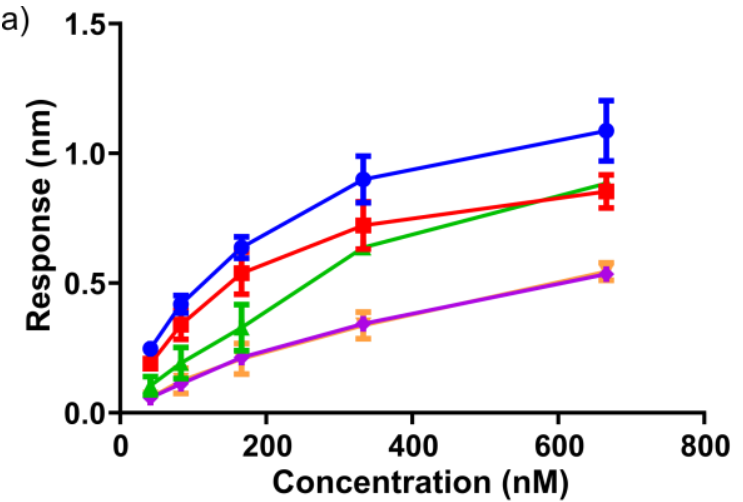

$\rightarrow$ Trastuzumab $8\left(K_{D}=1.75 \times 10^{-7} \mathrm{M}\right)$

$\Rightarrow$ Natively re-bridged $12\left(K_{D}=3.28 \times 10^{-7} \mathrm{M}\right)$

$\rightarrow$ Disulfide scrambled $13\left(\mathrm{~K}_{\mathrm{D}}=4.94 \times 10^{-7} \mathrm{M}\right)$

$\rightarrow$ Thiol capped (MAR 4) $14\left(K_{D}=7.89 \times 10^{-7} \mathrm{M}\right)$

$\rightarrow$ Thiol capped (MAR 8) $15\left(\mathrm{~K}_{\mathrm{D}}=4.50 \times 10^{-6} \mathrm{M}\right)$
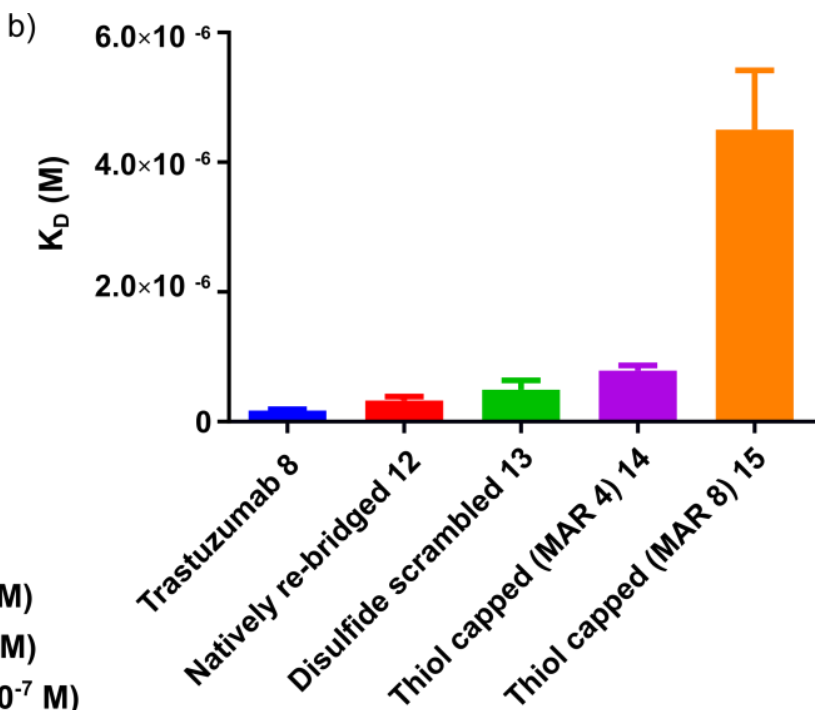

c)

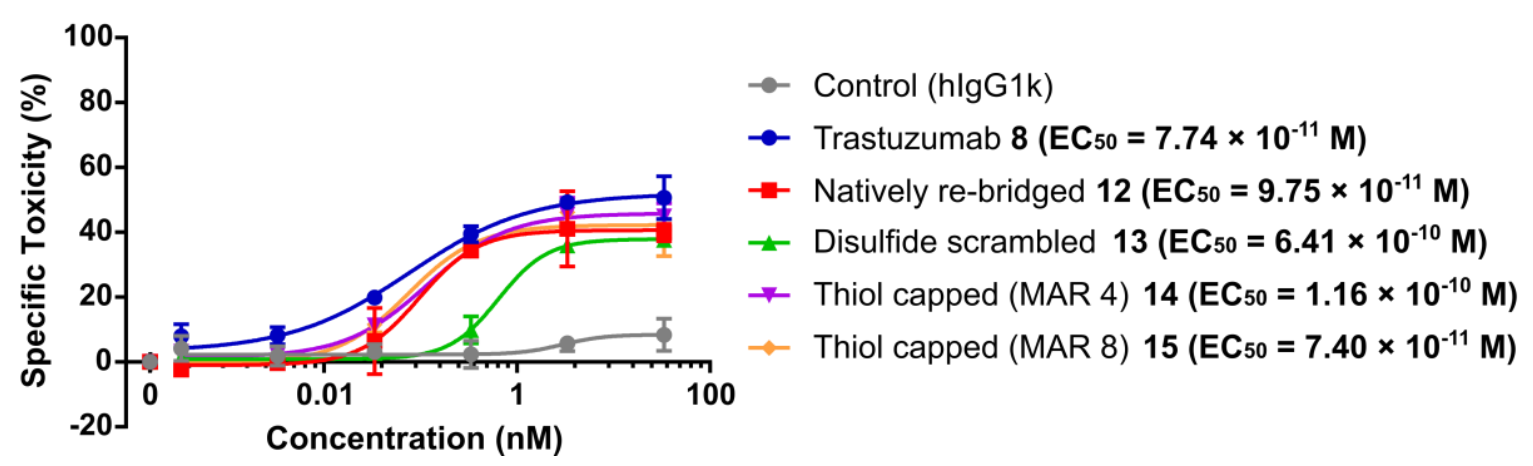

Figure 4 - Trastuzumab - FcRn BLI kinetics and ADCC cell kill assay summary. a) Maximum response at varying concentrations of Trastuzumab (8)/Trastuzumab conjugates (12-15). b) Average

Trastuzumab (8)/Trastuzumab conjugates (12-15) - CD16a $K_{D}$ values (see Table S5 in Supporting Information for details). c) ADCC dose response curves for Trastuzumab (8)/Trastuzumab conjugates

(12-15) with $\mathrm{EC}_{50}$ values.

To confirm the significance of the $C D 16 a K_{D}$ values obtained in the BLI assays, the synthesised conjugates were also subjected to an ADCC cell killing assay. BT-474 cells were incubated with each of the synthesised conjugates before adding CD16a+ NK-92 cells. Surprisingly, the conjugate repeatedly showing the least cell kill was the disulfide scrambled species $\mathbf{1 3}$. As this did not align with the trends observed in CD16a kinetics, it was anticipated this type of modification confers an unfavourable change in IgG1 interactions outside of the tested kinetic model (immobilised CD16atrastuzumab). Despite a significant increase in $K_{D}$ for some conjugates, all other tested species showed little decrease in ADCC activity. Whilst kinetic data is not always a good representation of what to expect in ADCC assays ${ }^{38}$, it is evident all types of disulfide modification affected either trastuzumab CD16a kinetics (thiol capped conjugates 14 and 15), or ADCC activity (disulfide scrambled conjugate 13) - with the exception of the natively re-bridged species $\mathbf{1 2 .}$

\section{Additional biophysical assays}

In addition to the results on IgG1 Fc function, a general biophysical profile was sought for each of the synthesised conjugates. In addition to native trastuzumab 8, the disulfide modified conjugates (1215) were compared in terms of antigen-binding, thermal stability and aggregation. These 
experiments were designed to provide a deeper understanding of the total influence that inter-chain covalent linkage integrity may have on other relevant antibody characteristics.

\section{Thermal stability:}

In a comparative experiment, melting temperatures (i.e. $\mathrm{T}_{\mathrm{m}}$ values) were calculated for each of the trastuzumab conjugates by conducting a thermal shift assay. Generally, a trend in intact inter-chain covalent links was established as the thiol capped conjugates $\mathbf{1 4}$ and $\mathbf{1 5}$ showed a linear decrease in $T_{m}$. Pleasingly, the natively re-bridged conjugate $\mathbf{1 2}$ had minimal change to the $T_{m}$, when compared with the native antibody 8 (Table 1). Unexpectedly, the disulfide scrambled conjugate $\mathbf{1 3}$ had a comparable $T_{m}$ to the thiol capped conjugate (MAR 4) $\mathbf{1 4}$ despite having a large proportion of the interchain disulfides correctly re-bridged (as 14 exists as a mixture of conjugates). We postulate that this may be indicative of an unstable conformation adopted by re-bridging the IgG1 disulfides in a non-native conformation.

SEC-MALS:

Approximate masses and polydispersity of the conjugate samples were compared with the intention of quantifying the amount of aggregate present, after bioconjugate synthesis. All tested conjugates yielded an insignificant percentage of aggregate by SEC-MALS. The disulfide scrambled conjugate $\mathbf{1 3}$ showed the most aggregation, with $2 \%$ of the mass being polydisperse, although this level of aggregate is not thought to be significant enough to affect antibody function (Table 1). Notably, natively re-bridging the disulfide residues showed no impact in terms of tendency to aggregate (see Table S8 in Supporting Information).

Enzyme-linked Immunosorbent Assay (ELISA):

Human epidermal growth factor (HER2) affinity was also measured for the synthesised conjugates, to confirm antigen-binding activity. All conjugates showed little deviation from the native trastuzumab control $\mathbf{8}$, which performed as expected, in accordance with previously reported results (see Figure S16 in Supporting Information). ${ }^{21}$

Table 1 Summary of additional biophysical data depicting percentage of sample considered aggregate by polydispersity; Thermal shift assays melting temperature $\left(T_{m}\right)$ values; calculated $I_{50}$ vales for trastuzumab - HER2 ELISA.

\begin{tabular}{lrrr}
\hline Trastuzumab Conjugate & $\begin{array}{l}\text { Aggregate mass } \\
\text { fraction (\%) }\end{array}$ & $\mathrm{T}_{\mathrm{m}}$ Value $\left({ }^{\circ} \mathrm{C}\right)$ & HER 2 IC \\
& 0.5 & $85.3 \pm 0.06$ & $6.783 \times 10^{-10}$ \\
\hline Trastuzumab 8 & 0.9 & $84.6 \pm 0.62$ & $5.837 \times 10^{-10}$ \\
Natively re-bridged 12 & 2.2 & $81.8 \pm 0.24$ & $4.530 \times 10^{-10}$ \\
Disulfide scrambled 13 & 0.2 & $81.7 \pm 0.08$ & $5.419 \times 10^{-10}$ \\
Thiol capped (MAR 4) 14 & 0.7 & $78.5 \pm 0.49$ & $5.397 \times 10^{-10}$ \\
Thiol capped (MAR 8) 15 & & & \\
\hline
\end{tabular}

\section{Conclusion}

This work represents the first example of appraising the Fc function of typical IgG1 bioconjugates formed via interchain disulfide-based modification strategies (including re-bridging and nonrebridging methods) in a single study; thermal stability, aggregation and antigen-binding were also measured on the same conjugates. CD16a and FcRn interactions were chosen to quantify effects of disulfide modification on trastuzumab (i.e. a model IgG1), which was achieved by use of BLI assays, 
and subsequent in vitro assays (i.e. ADCC) where applicable. The natively re-bridged IgG1 conjugate performed exceedingly well with no apparent decrease in CD16a or FcRn binding affinity, as well as no significant decrease in ADCC activity, suggesting re-bridging in a native conformation has minimal effect on IgG1 Fc function. It was found that by re-bridging the disulfides in a non-native conformation, whilst not affecting CD16a kinetics, did negatively influence the ADCC activity of the conjugate. Conversely, fully reducing and capping the disulfides of trastuzumab significantly affected the CD16a kinetics but did not yield significantly different ADCC results from the trastuzumab control. Further biophysical analysis of these conjugates (aggregation tendencies, thermal stability, antigen-binding) definitively showed that natively re-bridging the disulfide residues was the only modification strategy to have no negative impact on biophysical profile relative to the native antibody. This study therefore highlights the importance of the nature of disulfide modification on various properties and that natively re-bridging the disulfides appears to be the best way to retain the key properties of the native antibody. Moreover, through publishing the complexity of these secondary IgG1 mechanisms in a single study (e.g. CD16a binding is not necessarily correlated to $A D C C$ activity), it is envisaged that more efforts will be stimulated into understanding the relationship between antibody conjugation and Fc interactions.

\section{Conflicts of interest}

There are no immediate conflicts to declare, but we make clear that J.R.B. and V.C. are Directors of the spin-out ThioLogics.

\section{Acknowledgements}

We gratefully acknowledge the EPSRC (CASE Award with LifeArc, 173621) for funding CB, the EPSRC (EP/M01792X/1) for funding A.M., and the Leverhulme Trust (RPG-2017-288, 176274) for funding RS. We also acknowledge the UCL Chemistry Mass Spectrometry (MS) Facility (Dr Kersti Karu), and the EPSRC UK National MS Facility (Swansea).

\section{Supporting Information}

The Supporting Information is available free of charge on the ACS Publications website. 


\section{$\underline{\text { References }}$}

(1) Krall, N., da Cruz, F. P., Boutureira, O., Bernardes, G. J. L. (2016) Site-Selective ProteinModification Chemistry for Basic Biology and Drug Development. Nat. Chem. 8, 103-113.

(2) Spicer, C. D., Davis, B. G. (2014) Selective Chemical Protein Modification. Nat. Commun. 5, $1-14$.

(3) Alley, S. C., Okeley, N. M., Senter, P. D. (2010) Antibody-drug Conjugates: Targeted Drug Delivery for Cancer. Curr. Opin. Chem. Biol. 14, 529-537.

(4) Chari, R. V. J., Miller, M. L., Widdison, W. C. (2014) Antibody-Drug Conjugates: An Emerging Concept in Cancer Therapy. Angew. Chemie Int. Ed. 53, 3796-3827.

(5) Perez, H. L., Cardarelli, P. M., Deshpande, S., Gangwar, S., Schroeder, G. M., Vite, G. D., Borzilleri, R. M. (2014) Antibody-Drug Conjugates: Current Status and Future Directions. Drug Discov. Today 19, 869-881.

(6) Godwin, C. D., Gale, R. P., Walter, R. B. (2017) Gemtuzumab Ozogamicin in Acute Myeloid Leukemia. Leukemia 31, 1855-1868.

(7) LoRusso, P. M., Weiss, D., Guardino, E., Girish, S., Sliwkowski, M. X. (2011) Trastuzumab Emtansine: A Unique Antibody-Drug Conjugate in Development for Human Epidermal Growth Factor Receptor 2-Positive Cancer. Clin. Cancer Res. 17, 6437-6447.

(8) Lamb, Y. N. (2017) Inotuzumab Ozogamicin: First Global Approval. Drugs 77, 1603-1610.

(9) Katz, J., Janik, J. E., Younes, A. (2011) Brentuximab Vedotin (SGN-35). Clin. Cancer Res. 17, 6428-6436.

(10) Chudasama, V., Maruani, A., Caddick, S. (2016) Recent Advances in the Construction of Antibody-Drug Conjugates. Nat. Chem. 8, 114-119.

(11) Szijj, P. A., Bahou, C., Chudasama, V. (2018) Minireview: Addressing the Retro-Michael Instability of Maleimide Bioconjugates. Drug Discov. Today Technol. 30, 27-34.

(12) Wang, L., Amphlett, G., Blättler, W. A., Lambert, J. M., Zhang, W. (2005) Structural Characterization of the Maytansinoid-Monoclonal Antibody Immunoconjugate, HuN901DM1, by Mass Spectrometry. Protein Sci. 14, 2436-2446.

(13) Hamblett, K. J. (2004) Effects of Drug Loading on the Antitumor Activity of a Monoclonal Antibody Drug Conjugate. Clin. Cancer Res. 10, 7063-7070.

(14) Junutula, J. R., Flagella, K. M., Graham, R. A, Parsons, K. L., Ha, E., Raab, H., Bhakta, S., Nguyen, T., Dugger, D. L., Li, G. et al. (2010) Engineered Thio-Trastuzumab-DM1 Conjugate with an Improved Therapeutic Index to Target Human Epidermal Growth Factor Receptor 2Positive Breast Cancer. Clin. Cancer Res. 16, 4769-4778.

(15) Junutula, J. R., Raab, H., Clark, S., Bhakta, S., Leipold, D. D., Weir, S., Chen, Y., Simpson, M., Tsai, S. P., Dennis, M. S. et al. (2008) Site-Specific Conjugation of a Cytotoxic Drug to an Antibody Improves the Therapeutic Index. Nat. Biotechnol. 26, 925-932.

(16) McDonagh, C. F., Turcott, E., Westendorf, L., Webster, J. B., Alley, S. C., Kim, K., Andreyka, J., Stone, I., Hamblett, K. J., Francisco, J. A. et al. (2006) Engineered Antibody-Drug Conjugates with Defined Sites and Stoichiometries of Drug Attachment. Protein Eng. Des. Sel. 19, 299- 
307.

(17) Moura, A., Savageau, M. A., Alves, R. (2013) Relative Amino Acid Composition Signatures of Organisms and Environments. PLoS One 8, e77319.

(18) Hamblett, K. J., Senter, P. D., Chace, D. F., Sun, M. M. C., Lenox, J., Cerveny, C. G., Kissler, K. M., Bernhardt, S. X., Kopcha, A. K., Zabinski, R. F. et al. (2004) Effects of Drug Loading on the Antitumor Activity of a Monoclonal Antibody Drug Conjugate. Clin. Cancer Res. 10, 70637070.

(19) Dall'Acqua, W. F., Cook, K. E., Damschroder, M. M., Woods, R. M., Wu, H. (2006) Modulation of the Effector Functions of a Human IgG1 through Engineering of Its Hinge Region. J. Immunol. 177, 1129-1138.

(20) Lee, M. T. W., Maruani, A., Baker, J. R., Caddick, S., Chudasama, V. (2016) Next-Generation Disulfide Stapling: Reduction and Functional Re-Bridging All in One. Chem. Sci. 7, 799-802.

(21) Bahou, C., Richards, D. A., Maruani, A., Love, E., Javaid, F., Caddick, S., Baker, J., Chudasama, V. (2018) Highly Homogeneous Antibody Modification through Optimisation of the Synthesis and Conjugation of Functionalised Dibromopyridazinediones. Org. Biomol. Chem. 16, 13591366.

(22) Badescu, G., Bryant, P., Bird, M., Henseleit, K., Swierkosz, J., Parekh, V., Tommasi, R., Pawlisz, E., Jurlewicz, K., Farys, M. et al. (2014) Bridging Disulfides for Stable and Defined Antibody Drug Conjugates. Bioconjug. Chem. 25, 1124-1136.

(23) Robinson, E., Nunes, J. P. M., Vassileva, V., Maruani, A., Nogueira, J. C. F., Smith, M. E. B., Pedley, R. B., Caddick, S., Baker, J. R., Chudasama, V. (2017) Pyridazinediones Deliver Potent, Stable, Targeted and Efficacious Antibody-drug Conjugates (ADCs) with a Controlled Loading of 4 Drugs per Antibody. RSC Adv. 7, 9073-9077.

(24) Maruani, A., Smith, M. E. B., Miranda, E., Chester, K. A., Chudasama, V., Caddick, S. (2015) A Plug-and-Play Approach to Antibody-Based Therapeutics via a Chemoselective Dual Click Strategy. Nat. Commun. 6, 6645-6655.

(25) Lee, M. T. W., Maruani, A., Richards, D. A., Baker, J. R., Caddick, S., Chudasama, V. (2017) Enabling the Controlled Assembly of Antibody Conjugates with a Loading of Two Modules without Antibody Engineering. Chem. Sci. 8, 2056-2060.

(26) Shao, S., Tsai, M.-H., Lu, J., Yu, T., Jin, J., Xiao, D., Jiang, H., Han, M., Wang, M., Wang, J. (2018) Site-Specific and Hydrophilic ADCs through Disulfide-Bridged Linker and Branched PEG. Bioorg. Med. Chem. Lett. 28, 1363-1370.

(27) a) Schumacher, F. F., Nunes, J. P. M., Maruani, A., Chudasama, V., Smith, M. E. B., Chester, K. A., Baker, J. R., Caddick, S. (2014) Next Generation Maleimides Enable the Controlled Assembly of Antibody-drug Conjugates via Native Disulfide Bond Bridging. Org. Biomol. Chem. 12, 7261-7269.

b) Sun, S., Akkapeddi, P., Marques, M. C., Martínez-Sáez, N., Torres, V. M., Cordeiro, C., Boutureira, O., Bernardes, G. J. L. (2019) One-Pot Stapling of Interchain Disulfides of Antibodies Using an Isobutylene Motif. Org. Biomol. Chem. 17, 2005-2012.

(28) Guilliams, M., Bruhns, P., Saeys, Y., Hammad, H., Lambrecht, B. N. (2014) The Function of Fcy Receptors in Dendritic Cells and Macrophages. Nat. Rev. Immunol. 14, 94-108.

(29) Kuo, T. T., Baker, K., Yoshida, M., Qiao, S. W., Aveson, V. G., Lencer, W. I., Blumberg, R. S. (2010) Neonatal Fc Receptor: From Immunity to Therapeutics. J. Clin. Immunol. 30, 777-789. 
(30) Vidarsson, G., Dekkers, G., Rispens, T. (2014) IgG Subclasses and Allotypes: From Structure to Effector Functions. Front. Immunol. 5, 1-17.

(31) Teillaud, J.-L. (2001) Antibody-Dependent Cellular Cytotoxicity (ADCC). eLS 1-8.

(32) Verma, S., Miles, D., Gianni, L., Krop, I. E., Welslau, M., Baselga, J., Pegram, M., Oh, D.-Y., Diéras, V., Guardino, E. et al. (2012) Trastuzumab Emtansine for HER2-Positive Advanced Breast Cancer. N. Engl. J. Med. 367, 1783-1791.

(33) Dakshinamurthy, P., Mukunda, P., Prasad Kodaganti, B., Shenoy, B. R., Natarajan, B., Maliwalave, A., Halan, V., Murugesan, S., Maity, S. (2017) Charge Variant Analysis of Proposed Biosimilar to Trastuzumab. Biologicals 46, 46-56.

(34) Greene, M., Richards, D. A., Nogueira, J., Campbell, K., Smyth, P., Fernandez, M., Scott, C. J., Chudasama, V. (2018) Generating Next-Generation Antibody-Nanoparticle Conjugates through the Oriented Installation of Non-Engineered Antibody Fragments. Chem. Sci. 9, 7987.

(35) a) Buecheler, J. W., Winzer, M., Tonillo, J., Weber, C., Gieseler, H. (2018) Impact of Payload Hydrophobicity on the Stability of Antibody-Drug Conjugates. Mol. Pharm. 15, 2656-2664 b) Behrens, C. R., Ha, E. H., Chinn, L. L., Bowers, S., Probst, G., Fitch-Bruhns, M., Monteon, J., Valdiosera, A., Bermudez, A., Liao-Chan, S. et al. (2015) Antibody-Drug Conjugates (ADCs) Derived from Interchain Cysteine Cross-Linking Demonstrate Improved Homogeneity and Other Pharmacological Properties over Conventional Heterogeneous ADCs. Mol. Pharm. 12, 3986-3998.

c) Lyon, R. P., Bovee, T. D., Doronina, S. O., Burke, P. J., Hunter, J. H., Neff-Laford, H. D., Jonas, M., Anderson, M. E., Setter, J. R., Senter, P. D. (2015) Reducing Hydrophobicity of Homogeneous Antibody-Drug Conjugates Improves Pharmacokinetics and Therapeutic Index. Nat. Biotechnol. 33, 733-736.

(36) Mankarious, S., Lee, M., Fischer, S., Pyun, K. H., Ochs, H. D., Oxelius, V. A., Wedgwood, R. J. (1988) The Half-Lives of IgG Subclasses and Specific Antibodies in Patients with Primary Immunodeficiency Who Are Receiving Intravenously Administered Immunoglobulin. J. Lab. Clin. Med. 112, 634-640.

(37) Souders, C. A., Nelson, S. C., Wang, Y., Crowleya, A. R., Klempner, M. S., Thomas, W. (2015) A Novel in Vitro Assay to Predict Neonatal Fc Receptor-Mediated Human IgG Half-Life. MAbs 7, 912-921.

(38) Stavenhagen, J. B., Gorlatov, S., Tuaillon, N., Rankin, C. T., Li, H., Burke, S., Huang, L., Johnson, S., Bonvini, E., Koenig, S. (2007) Fc Optimization of Therapeutic Antibodies Enhances Their Ability to Kill Tumor Cells in Vitro and Controls Tumor Expansion in Vivo via Low-Affinity Activating Fcy Receptors. Cancer Res. 67, 8882-8890. 